\title{
MAGNETIC FIELD MEASUREMENT ON SUPERCONDUCTING MULTIPOLE WIGGLER WITH NARROW DUCT
}

\author{
T.C. Fan, F.Y. Lin, M.H. Huang, C.H. Chang, C.S. Hwang, NSRRC, Taiwan
}

\begin{abstract}
The first superconducting multipole wiggler with period length of $6.0 \mathrm{~cm}$ (SMPW6) of the National Synchrotron Radiation Research Center (NSRRC) is currently under construction. SMPW6 will be tested and installed at the RF cavity section of storage ring by the autumn of 2003. For quality control, the magnetic field is checked at three main phases according to the assembly process. To operate the field measurement in a narrow duct and at different temperature of duct at different phases, three small-gap measurement systems were specially designed. Not only the centerline field can be measured point-by-point but also the multi-pole field can be mapped. All the measurement systems have been carefully tested. The consideration of the measurement systems and preliminary measurement are shown in this paper.
\end{abstract}

\section{INTRODUCTION}

For a medium energy synchrotron radiation facility like NSRRC, the demand of $x$-ray has been increasing. After all the straight sections for insertion devices of storage ring have been occupied, NSRRC explores any possibilities to provide hard $\mathrm{x}$-ray to users. NSRRC has made a superconducting wavelength shifter (SWLS), with operation field of $6 \mathrm{~T}$, and tested it in even the injection section [1]. Next, in the residual space of the superconducting RF (SRF) cavity straight-sections, a superconducting multipole wiggler (SMPW6) will be installed (see Fig. 1)[2]. At this residual space of only $140.56 \mathrm{~cm}$ for SMPW6, 28 effective poles (32 total poles) with the periodic length of $6.0 \mathrm{~cm}$ will be created and a $3.2 \mathrm{~T}$ field will be excited. The SMPW6 is a cooperative project with Wang NMR Inc., US. To control the quality carefully, the magnetic field is to be checked at three main phases according to the assembly process.

The SMPW6 is designed to operate in liquid helium. To reduce the heat load of the $4.2 \mathrm{~K}$ cold mass, a thermal-shielding elliptic beam-duct of $1 \mathrm{~mm}$ thick with $77 \mathrm{~K}$ interception, and a vacuum barrier of $1 \mathrm{~mm}$ thick as thermal barrier are employed. As a result, the $12 \mathrm{~mm}$ cold beam gap and a cold iron pole separation of $18 \mathrm{~mm}$ are required. For final acceptance test, an extensive field measurement is affordable if operating at room temperature. Thus an additional flat warm duct is provided. The allowed vertical inner space is only 5.9 $\mathrm{mm}$. Because of the variant measurement conditions at different phases, in terms of limited space and space temperature, three small-gap measurement systems were specially designed. Other than centerline field, the multi-pole field can be mapped as well.

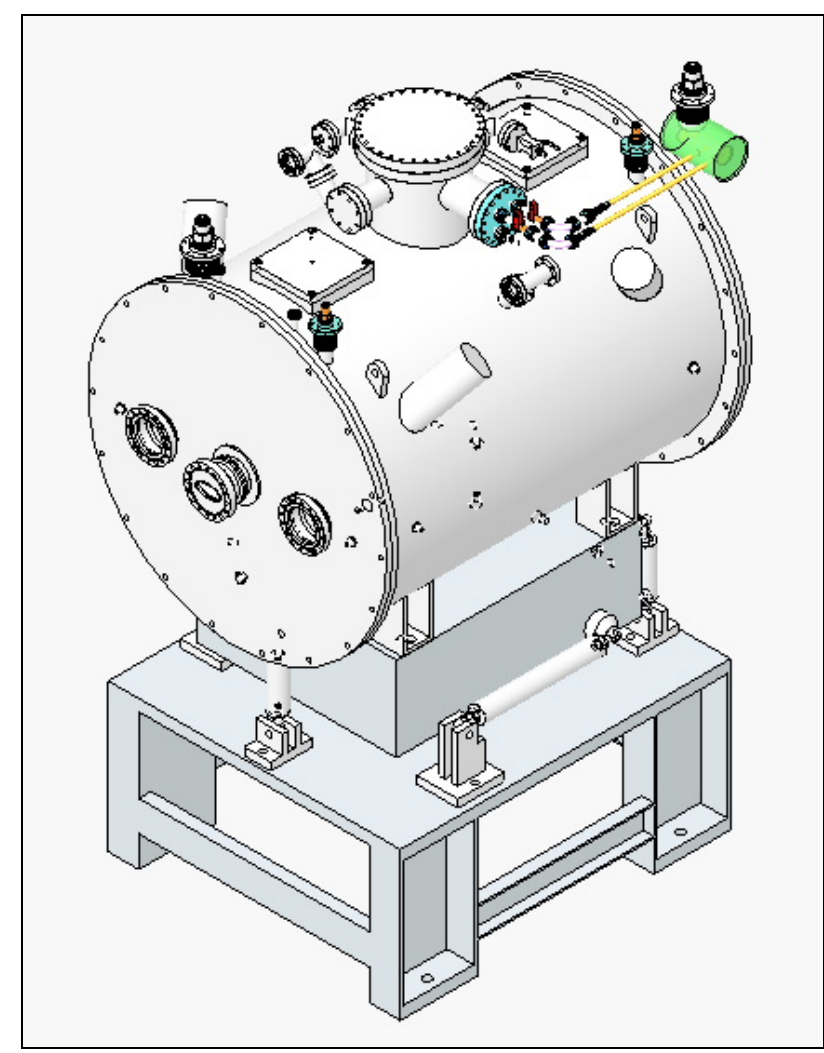

Figure 1: Schematic drawing of SMPW6.

At the first phase, a 5-pole prototype is tested at NSRRC. The newly built cryogenic plant will not be functional until the end of 2003 (actually the SWLS is cryogen-free and conduction-cooled by a 1.5-Watt GM-type cryo-cooler.). Therefore, the magnet is tested in a test tank cooled by a cryo-cooler. To operate under small heat-load budget, a measurement system featuring small-diameter pipe was proposed to provide a convenient room-temperature measurement. At this phase, the measurement is called project A.

While going to the second phase, all the magnetic poles have been fabricated and assembled. Before the magnet was enclosed into the cryostat, all the problems related to the magnet itself have to be clarified. The magnet is immersed in a dewar and a Hall probe capable of operating at liquid helium can be scanned in an easy rail. The measurement at this stage is named Project $B$.

At final phase, the magnet is assembled into a cryostat as a whole and an acceptance test begins. An extensive measurement is essential to characterize the magnetic performance for beam dynamics and spectrum output. A wide flat duct at room temperature is preferred. However wide duct corresponds to a big heat load. Project $\mathrm{C}$ covers the final measurement. 


\section{MEASUREMENT SYSTEMS}

Two categories of measurement system, namely Hall probe and stretched wire, are adopted in SMPW6 certification. The former presents different transportation method at each of three phases.

\section{Project $A$}

A tailored stage equipped a narrow cylinder tube (referred to as endo-chamber) provides low heat-leak as well as room-temperature space for measurement sensors. The magnet is mounted in a test chamber, then vacuumed barrier and cooled to $4.5 \mathrm{~K}$. A flat epi-chamber connected to $70 \mathrm{~K}$ interception to further reduce the heat load. Utilizing the endo-chamber, one can shuttle the Hall probe by a long strike $x-y$ stage or rotate the long loop of stretched wire to measure the point field or integral field. Fig. 2 shows a cartoon of Project A.

With the mounted bellow and small x-y stage, one can level the endo-chamber and move it horizontally to scan the field. As the bellow will absorb the tremendous atmosphere pressure, the small $x-y$ stage must be fastened well and the distortion of endo-chamber is to be cautious. The sag of endo-chamber owing to gravity needs suitable pads to compensate.

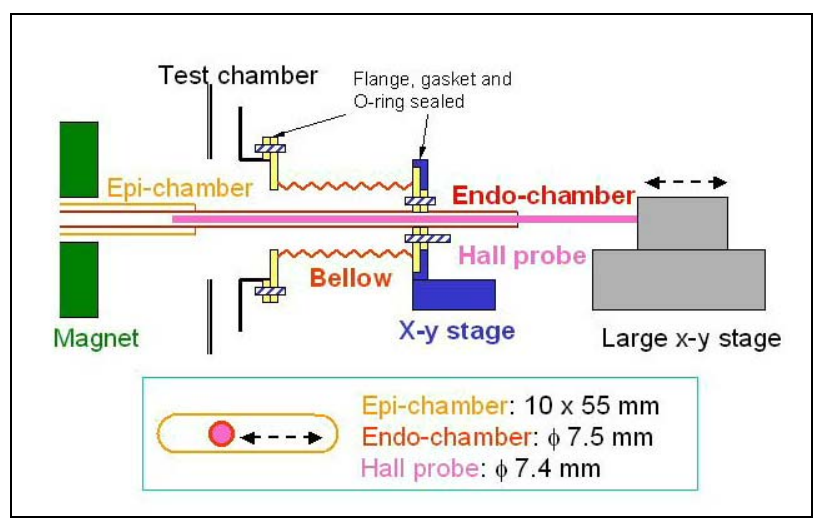

Figure 2: A cartoon of the measurement system Project A. Endo-chamber can move horizontally within the epi-chamber with the help of bellow. Large $x-y$ stage can shuttle the Hall probe along the endo-chamber to map the field.

\section{Project $B$}

The easiest way to map the field of the superconducting magnet is to immerse the magnet in liquid helium of dewar and then move a crogenic Hall probe vertically to measure the field point-by-point. To confine the probe without transverse position error, a precise rail structure guiding the probe is equipped. A stepping motor and screw bolt provide a precise longitudinal movement. In project B, a Lake Shore probe MCA-3160-WN, featuring wide operating temperature range ( $1.5 \mathrm{~K}$ to $350 \mathrm{~K})$, is used. To travel into a high dewar, a 60 " stem is chosen. A set of 3 parallel rails provide the hall probe the field mapping along $x=-20,0$ and $=20 \mathrm{~mm}$, so as to check the roll-off and quadrupole component. To reduce the boiling-off of the liquid helium, a transfer rail set made of FRP and radiation shielding on top of the magnet are equipped. The vertical configuration avoids the sag of horizontal hall stem. Due to low temperature, the rail bending and reduction of tolerance of space between probe head and rail have to be taken care. Fig. 3 shows the project $B$ system under assembling and testing.

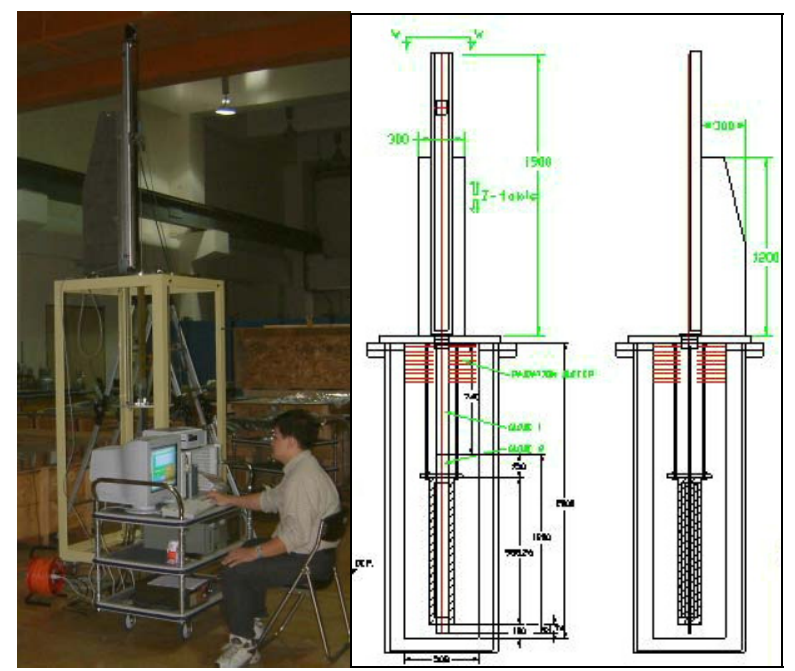

Figure 3: The measurement system Project $\mathrm{B}$ under assembly (left photo) and merged into dewar and core magnet (right drawing).

The preliminary measured field in vertical dewar was shown in Fig. 4 and 5. The measured field on-axis shows that the second field integral is quite good without any correction.

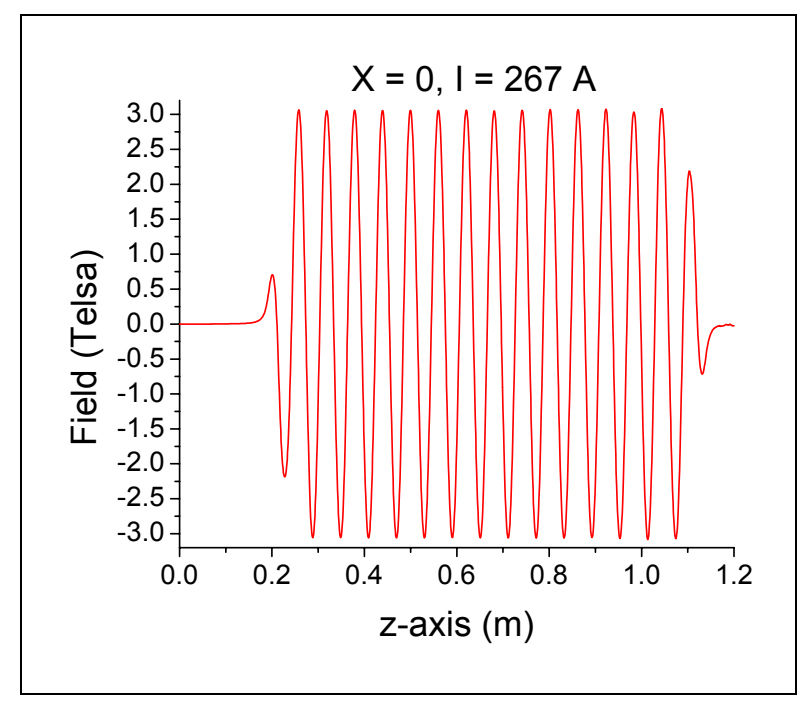

Figure 4: Magnetic field measured in Project B. 


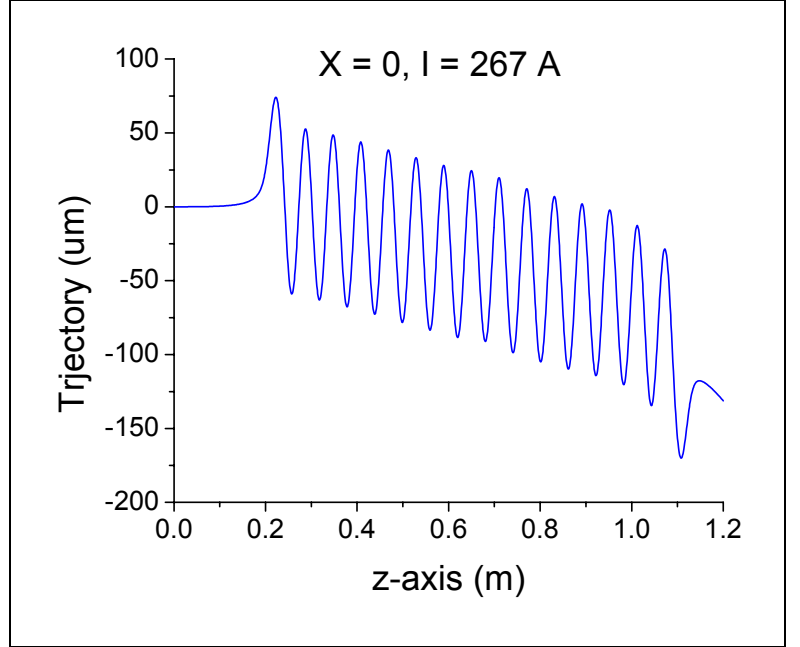

Figure 5: The centerline trajectory calculated from the second integral field of Project B.

\section{Project $C$}

The magnetic field measurement system is operated at room temperature at the final phase. A flat warm duct with a space of $50 \mathrm{~mm}$ width while $5.9 \mathrm{~mm}$ high is required. In such a narrow vertical space, the position of the Hall probe on the end of a thin stem is not predicable, due to the sag and the friction with the duct. Instead, a Hall probe is assembled on a taut strip of FRP tensioned by a strong bow of I-beam. The I-beam is rested on an $\mathrm{x}-\mathrm{y}$ stage to map the periodical field profile point-by-point. The bow beam with I shape can facilitate the weight balance and alignment. With the help of a strong back, the moving track of the $\mathrm{x}-\mathrm{y}$ stage achieves the lowest deformation. The FRP strip instead of metal one can support the on-the-fly measurement without worry of eddy current. Fig. 6 and 7 show the configuration of the Hall probe system in Project C.

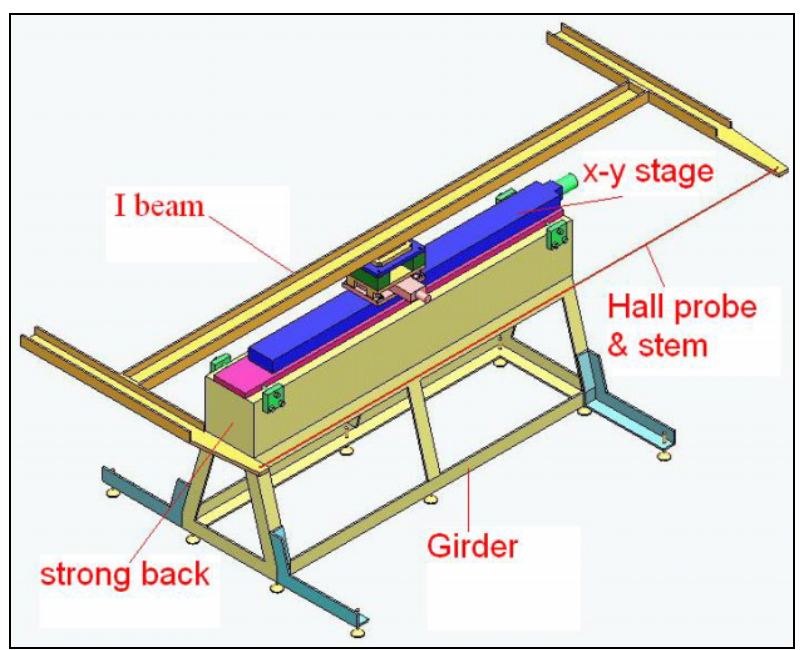

Figure 6: A cartoon of the I-beam hall probe measurement system used in Project C.

On the other hand, to fulfill the extensive measurement, the horizontal space is wide. Wide duct means big heat leak. Cryo-cooler cannot afford such heat load and a large consumption of liquid helium is expected. Meanwhile a big temperature gradient occurred during test. A small air injection system and a Senstron YM12-4 probe with well-defined temperature coefficient are used to control the temperature range and calculate the correction value. Moreover, the water vapor will quickly condense and freeze on the inner surface of duct and shrink the space. Frequent clearance of ice is necessary during the measurement. In addition, the drift due to the bending of signal line during stage movement is well calibrated. The final reproducibility is within \pm 2 Gauss.

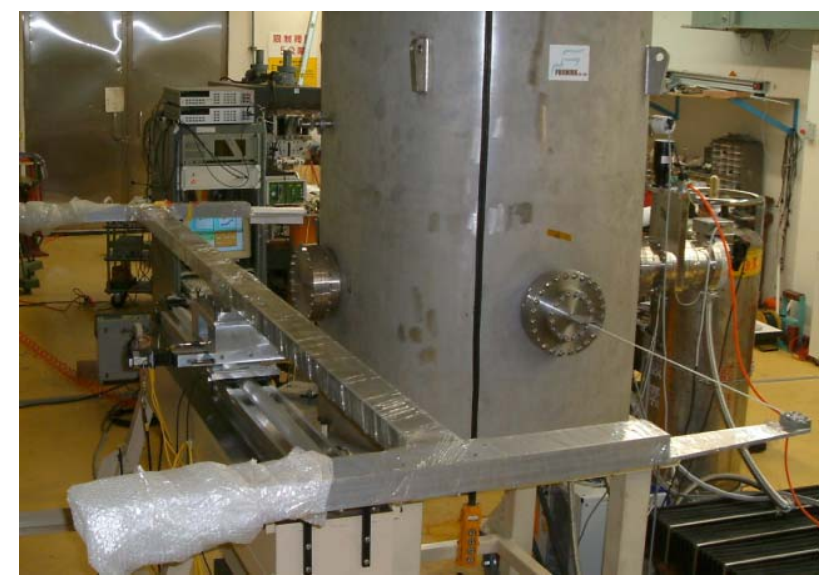

Figure 7: I-beam system under testing.

The stretched-wire technique is also used to measure the mulipole components of 1 st and 2 nd integral field directly with horizontal range of $\pm 20 \mathrm{~mm}$ (see Fig. 8).

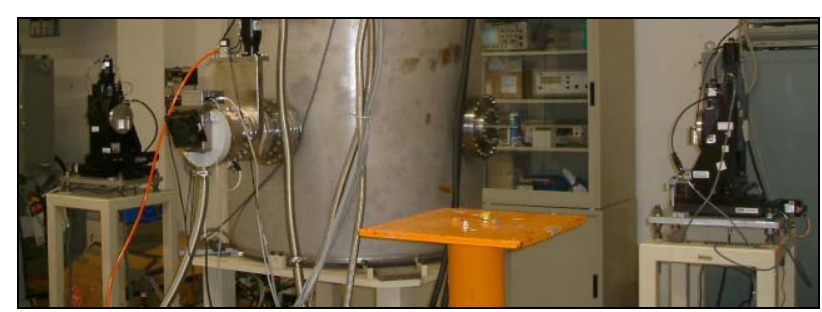

Figure 8: A stretched wire measurement system under test for Project C.

\section{CONCLUSION}

The strategy of the measurement system for SMPW6 at different phases was reported. A solution of small-gap measurement on low- temperature magnet appeared feasible. From the preliminary measurement, the SMPW6 has good magnetic performance in terms of beam dynamics.

\section{REFERENCES}

[1] C. S. Hwang, et al., IEEE Trans. Appl. Supercond., vol. 12 , no. 1, 686 (2002).

[2] C. S. Hwang, et al., will be published in IEEE Trans. Appl. Supercond. (2003). 